\title{
Knowledge, Awareness and Attitude of Predisposing Factors of Fatty Liver Among the General Population
}

\author{
Kaviya. L' ${ }^{1}$ Dr. M. P. Brundha ${ }^{2 *}$ and Dr.Preejitha.V.B ${ }^{3}$ \\ ${ }^{1}$ Undergraduate Student Saveetha Dental College, Saveetha Institute of Medical and \\ Technical Sciences, Saveetha University. Chennai-600077, Tamil Nadu, India. \\ ${ }^{2}$ Associate professor, Department of Pathology Saveetha Institute of Medical and Technical Sciences, \\ Saveetha University, 162, Poonamalle High Road, Chennai-600077, Tamil Nadu, India. \\ ${ }^{3}$ Department of Pathology Saveetha Dental College, Saveetha Institute of Medical and Technical Sciences \\ Saveetha University. Chennai-600077, Tamil Nadu, India.
}

\begin{abstract}
The aim of my study is to know the attitude and awareness of predisposing factors of fatty liver disease. Fatty liver disease is also known as hepatic steatosis, where excess fat builds up in the liver. There are two types of fatty liver disease alcoholic and nonalcoholic fatty disease. Alcoholic fatty liver disease is due to the heavy use of alcohol. Globally, approximately 2 million people die of the liver disease each year, and up to 50\% of mortality with cirrhosis is attributable to alcohol. Nonalcoholic fatty liver disease is due to excess fat build-up in the liver with insulin resistance due to causes other than alcohol use. NAFLD is a liver disease associated with obesity, insulin resistance, type 2 diabetes mellitus, hypertension, hyperlipidemia, and metabolic syndrome. A study was conducted among 100 people by preparing a set of questions through an online survey method. The present study shows fatty liver diseases are caused by lifestyle changes, malnutrition and it can occur in nonalcoholic people. Fatty liver disease is a growing global health problem, affecting almost a quarter of the world's population. Fatty liver diseases are closely associated with obesity and metabolic syndrome, it is worrisome that its prevalence may soon reach epidemic proportions worldwide.
\end{abstract}

KEY WORDS: FATTY LIVER DISEASE, OBESITY, ALCOHOL.

\section{INTRODUCTION}

Fatty liver disease is also known as hepatic steatosis, where excess fat builds up in the liver. There are two types of fatty liver disease alcoholic and nonalcoholic fatty disease(Basra and Anand, 2011). Alcoholic fatty liver disease is due to the heavy use of alcohol. The liver breaks down most of the alcohol in the body and it can be

\section{ARTICLE INFORMATION}

*Corresponding Author: brundha.sdc@saveetha.com Received 10th Augsut 2020 Accepted after revision 27th Sep 2020 Print ISSN: 0974-6455 Online ISSN: 2321-4007 CODEN: BBRCBA

Thomson Reuters ISI Web of Science Clarivate Analytics USA and Crossref Indexed Journal

\section{Clarivate
Analytics}

NAAS Journal Score 2020 (4.31) SJIF: 2020 (7.728)

A Society of Science and Nature Publication,

Bhopal India 2020. All rights reserved.

Online Contents Available at: http//www.bbrc.in/

Doi: http://dx.doi.org/10.21786/bbrc/13.8/111 removed from the body. During this process of breaking alcohol, it generates harmful substances. These harmful substances can damage liver cells ,weaken the body's natural defenses and promote inflammation. Alcoholic fatty liver disease is the earliest stage of alcohol-related liver disease. The next stages are alcoholic hepatitis and cirrhosis(Osna, Donohue, and Kharbanda, 2017). Globally, approximately 2 million people die of the liver disease each year, and up to 50\% of mortality with cirrhosis is attributable to alcohol.

The alcohol-related liver disease represents one of the top 30 causes of death in recent studies on the global burden of disease. In 2010, the worldwide rate of alcoholattributable cirrhosis death was 7.2 deaths per 100,000 people (4.6 in females and 9.7 in males) (Thursz et al., 2019). Nonalcoholic fatty liver disease is due to excessive fat build-up in the liver with insulin resistance due to

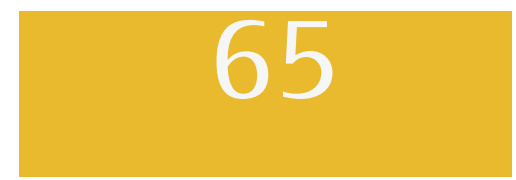


causes other than alcohol use. There are two types; non-alcoholic fatty liver (NAFL) and non-alcoholic steatohepatitis (NASH), with the latter also including liver inflammation(Younossi, 2019),('National Institute of Diabetes and Digestive and Kidney Diseases (NIDDK)', 2008).

NAFLD is a liver disease associated with obesity, insulin resistance, type 2 diabetes mellitus, hypertension, hyperlipidemia, and metabolic syndrome.NAFLD leading to liver fibrosis, cirrhosis, hepatocellular carcinoma, and liver transplantation. All of these complications of NASH can pose significant health, economic, and patientexperience burdens to the patients, their families, and society(Lieber, 2004)

People with fatty liver disease have no or few symptoms. Occasionally there may be tiredness or pain in the upper right side of the abdomen (Friedman et al., 2018). The major predisposing factors of fatty liver disease are alcohol (during the metabolism of alcohol in the liver it produces toxic metabolites like alcohol)(Angulo, 2002), metabolic like glycogen storage disease, Weber Christian disease, acute fatty liver of pregnancy, lipodystrophy (Bayard, Holt, and Boroughs, 2006), diet composition and quantity in particular omega- 6 fatty acid lipids and fructose sugar, has an important role in disease progression from NAFL to NASH and fibrosis. Choline deficiency can lead to the development of NAFLD(Leermakers et al., 2015). Drugs and toxins like diltiazem, expired tetracycline, highly active antiretroviral therapy, glucocorticoids, tamoxifen, environmental hepatotoxins, other celiac diseases, inflammatory bowel disease, HIV, hepatitis C (especially genotype 3), and alpha 1-antitrypsin deficiency causes fatty liver(Osman, Osman, and Ahmed, 2007), (Marciano, Savoia, and Vajro, 2016).

Two genetic mutations for NAFLD susceptibility have been identified and validated in large cohorts: the nonsynonymous single-nucleotide polymorphisms (SNPs) in PNPLA3 and TM6SF2, as they have been shown to correlate with NAFLD presence and severity, but their roles for diagnosis remain unclear. Although NAFLD has a genetic component, the American Association for the study of liver disease does not recommend screening family members as there is not enough confirmation of heritability, although there is some evidence from familial aggregation and twin studies(Wong et al., 2018)Fatty liver disease can develop into liver cancer or fibrosis. Less than $10 \%$ of people with cirrhosis alcoholic fatty liver disease will develop hepatocellular carcinoma (Singh et al., 2015)

The diagnosis of fatty liver disease is identified by increased transaminases, especially ALT. The ratio of ALT/ AST is usually less than 1 but it is increased for the fatty liver diseased people. It is found during a health check-up or during the study of some other manifestation of the metabolic syndrome. Diagnosis may also be suspected by the accidental discovery of hepatomegaly or a radiological test carried out for another reason showing suggestive changes of fatty liver (Pagano, 2002).
Although the final diagnosis should be confirmed by liver biopsy(Chalasani et al., 2018).

The treatment of the fatty liver disease depends on various factors, causes, etc that not all are identified. In more serious cases, medications that decrease insulin resistance, hyperlipidemia, and those that induce weight loss such as bariatric surgery as well as Vitamin E have been shown to improve or resolve liver function(Caballería et al., 2008). Fatty liver is an ever more prevalent disease involving 17 to 33\% of the general population according to the different series and is increasing with the higher incidence of obesity(Bedogni et al., 2005).

\section{MATERIAL AND METHODS}

Sample Selection: A sum of 100 people of the general population were randomly selected from the university as a participant in the survey. The data collection questionnaire was developed after reviewing various similar literature .

Inclusion and Exclusion Criteria: Inclusion criterion set was the general population who were willing to attend the survey. Exclusion criteria was set for people who were not willing to participate.

Sampling Method: In the present study, the sampling method used is a random sampling method.

Data Collection and Tabulation: The questionnaire which was taken on a survey planet concerns about 15 questions mainly based on the concept of fatty liver. Their responses were entered into the excel sheets and then tabulation of the data finally and the question comparison was done. The representation of the data is through the bar graph.

Statistical Analysis: The statistical software used IBM SPSS V22.The statistical test used is Chi square test ( $p$ value). Type of analysis used were descriptive analysis,demographic data.

\section{RESULTS AND DISCUSSION}

The study shows (fig 1) 83\% of people heard about fatty liver, (fig 2) 59\% of people thought fatty liver diseases are hereditary. Kawaguchi and colleagues focused on establishing the genetic risk in Asian-descent patients(Kawaguchi et al., 2012). (Fig 3)70\% of people think fatty liver diseases were preventable,(fig 4) $84 \%$ thought treatment available for fatty liver disease, (fig 5) 67\% aware of fatty liver diseases are occur in nonalcoholic people, (fig 6) $82 \%$ thought fatty liver diseases are curable, ( fig 7) 75\% of people thought fatty liver disease cause serious health problems, (fig 8) 54\% of people not known fatty liver is linked with diabetes. $\mathrm{Lu}$ et al. reported the prevalence of NAFLD in type 2 diabetes mellitus patients was significantly associated with elevated ALT (Lu et al., 2009). (fig 10 ) 62\% of people are aware that it is linked with obesity and Bp, (fig 12 ) 54\% thought fatty liver is linked with hepatitis. 
Concurrent hepatitis $\mathrm{C}$ infection and steatosis have been associated with increased risk of disease progression. Genotype 3 is strongly associated with the presence of hepatic steatosis independent of sex, body mass index, and alcohol consumption. Current research suggests that one possible mechanism for hepatic steatosis in HCV is binding of the viral $\mathrm{X}$ gene product to LXR-alpha and upregulation of the transcription factor SREBP1c (sterol regulatory element-binding protein 1c)(Brown et al., 2005).

Figure 1: Pie chart represents the percentage of people heard about fatty liver. Red colour in the pie chart denotes"Yes" and blue colour denotes "No". A higher percentage of responses were Yes (83\%).

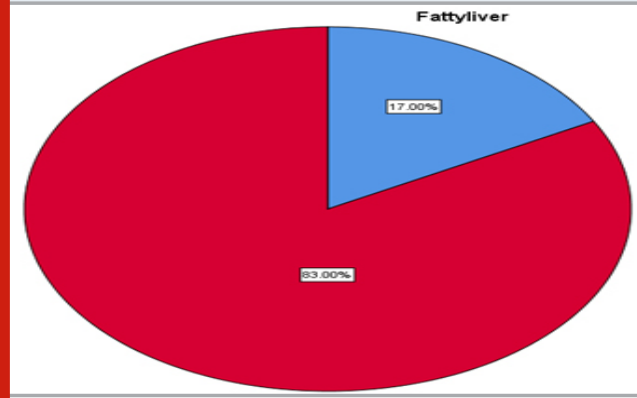

Figure 2: Pie chart represents the percentage of people thought fatty liver disease was hereditary. Red colour in the pie chart denotes"Yes" and blue colour denotes "No". Higher percentage of responses were Yes (59\%).

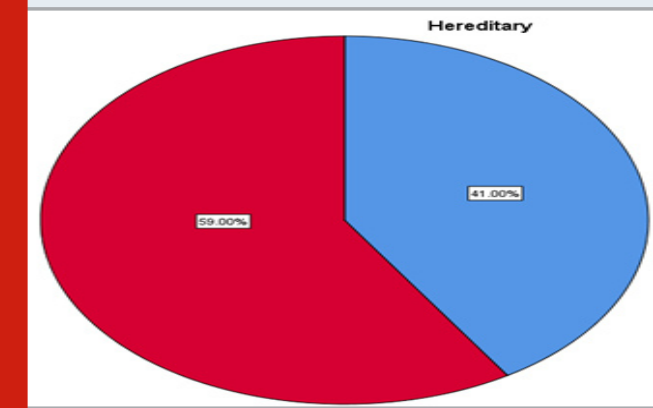

Figure 3: Pie chart represents the percentage of people thought about fatty liver diseases were preventable. Red colour in the pie chart denotes"Yes" and blue colour denotes "No". Higher percentage of responses were Yes (70\%).

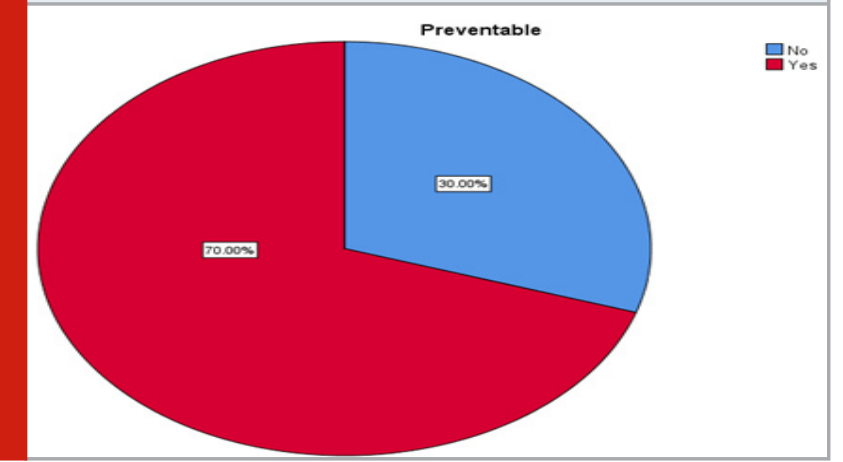

Figure 4: Pie chart represents the percentage of people thought about the treatment available for fatty liver diseases. Red colour in the pie chart denotes"Yes" and blue colour denotes "No". Higher percentage of responses were Yes $(84 \%)$.

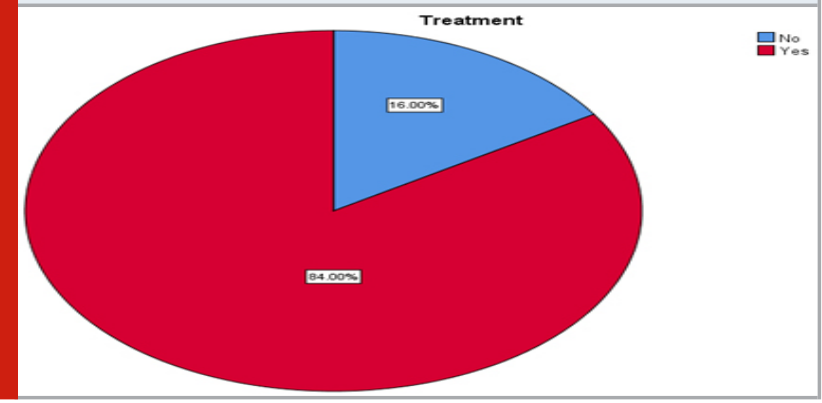

Figure 5: Pie chart represents the percentage of awareness about fatty liver diseases in nonalcoholic people. Red colour in the pie chart denotes"Yes" and blue colour denotes "No". Higher percentage of responses were Yes (67\%).

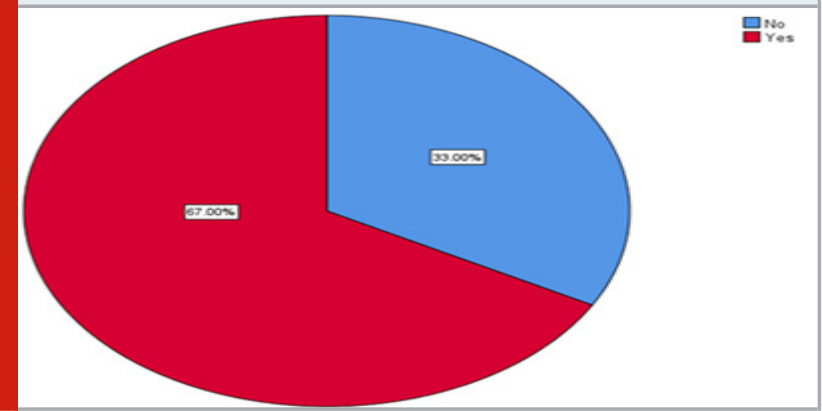

Figure 6: Pie chart represents the percentage of people thought fatty liver diseases are curable. Red colour in the pie chart denotes"Yes" and blue colour denotes "No". Higher percentage of responses were Yes (82\%).

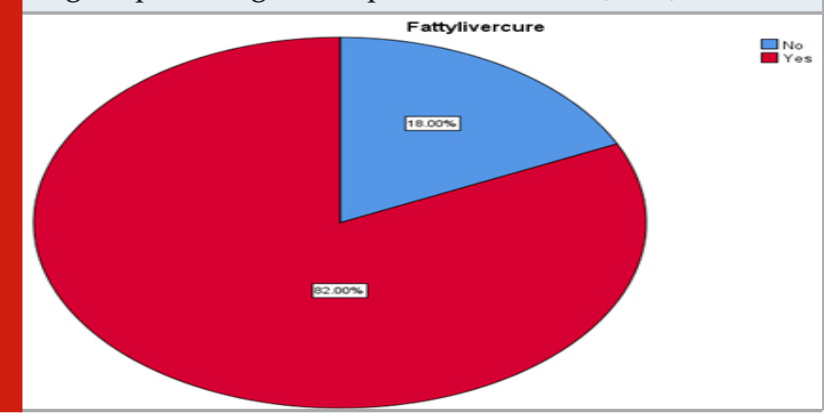

(Fig 13 ) 72\% of people were aware of lifestyle changes causing fatty liver, (fig 15 ) 60\% of people know fatty liver diseases are caused by medications,( fig 17 ) 61\% of people thought fatty liver disease is linked with PCOS. Few interventional data exist for NAFLD in PCOS patients. There is one case with a response to diet, moderate weight loss, and exercise with an improvement in her histology findings at a post-treatment liver biopsy(Kneeman, Misdraji, and Corey, 2012). (Fig 18 )60\% are aware that it is caused by malnutrition. 
Figure 7: Pie chart represents the percentage of people thought fatty liver diseases cause serious health problems. Red colour in the pie chart denotes"Yes" and blue colour denotes "No". Higher percentage of responses were Yes (75\%).

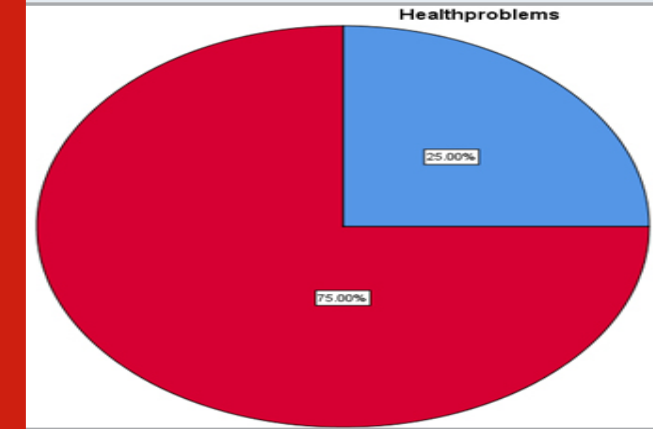

Figure 8: Pie chart represents the percentage of people thought fatty liver was linked with diabetes. Red colour in the pie chart denotes "Yes " and blue colour denotes "No". Higher percentage of responses were No (54\%).

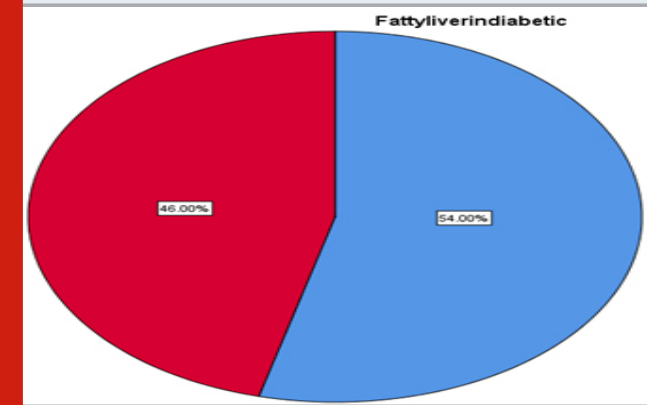

Figure 9: Bar chart showing the comparison of responses based on the question "Do you think fatty liver is linked with diabetes" between male and female. The $\mathrm{X}$-axis represents the sex and $\mathrm{Y}$-axis represents number of participants of which red colour denotes "Yes" and blue colour denotes "No" .Majority of the males thought fatty liver is not linked with diabetes.However the difference is not statistically significant (Chi square value -0.251 , p value $=0.616$ ( $>0.5$ - indicating statistically not significant).

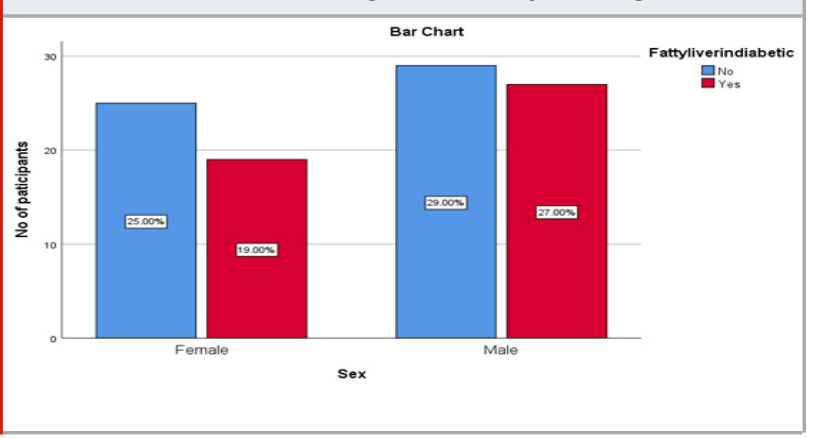

The cytosolic concentration of choline, a critical nutrient for lipoprotein secretion, also decreases, promoting lipid storage in hepatocytes(Hu et al., 2018). Our results demonstrated that fatty liver diseases are strongly associated with metabolic activities such as higher body fat, obesity, hyperlipidemia, and impaired fasting glucose(Dyson, Anstee, and McPherson, 2015). Lifestyle interventions aimed at weight loss and increased activity are essential for all patients with fatty liver disease and if sustained is effective in the treatment of fatty liver disease. For patients who develop advanced disease, they require surveillance for and management of the complications of cirrhosis (HCC, varices, osteoporosis) (Söderberg et al., 2010).

Figure 10: Pie chart represents the percentage of people thought fatty liver diseases were linked with $\mathrm{Bp}$ and obesity. Red colour in the pie chart denotes"Yes" and blue colour denotes "No". Higher percentage of responses were Yes $(62 \%)$.

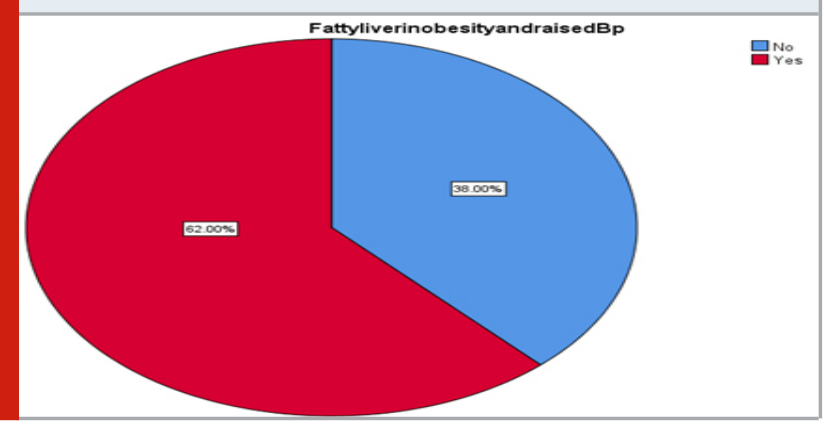

Figure 11: Bar chart showing the comparison of responses based on the question "Do you think obesity and raised $\mathrm{Bp}$ is linked with fatty liver" between male and female. The $\mathrm{X}$-axis represents the sex and $\mathrm{Y}$-axis represents number of participants of which red colour denotes "Yes" and blue colour denotes "No".Majority of the males thought fatty liver is thought fatty liver was linked with obesity and Bp.However the difference is not statistically significant (Chi square value $-1.274, p$ value $=0.259$ ( > 0.5 - indicating statistically not significant).

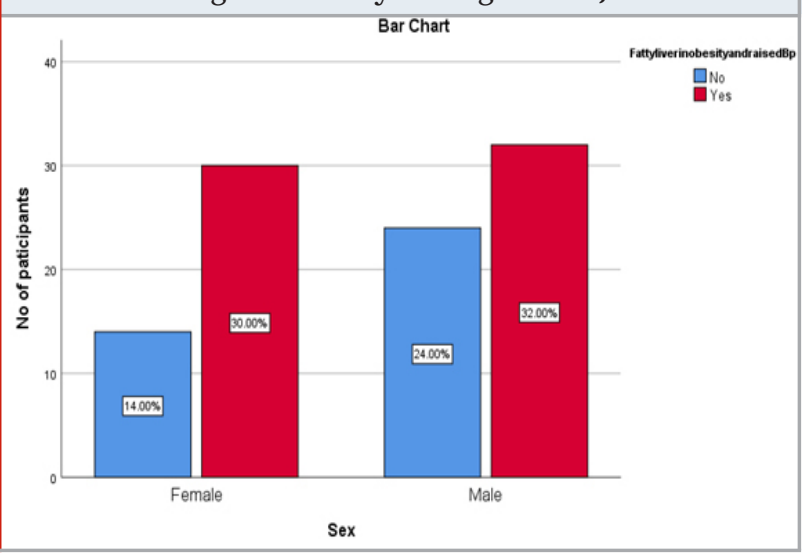


Figure 12: Pie chart represents the percentage of people thought fatty liver diseases were thought fatty liver is linked with hepatitis.Red colour in the pie chart denotes"Yes" and blue colour denotes "No". Higher percentage of responses were Yes (54\%).

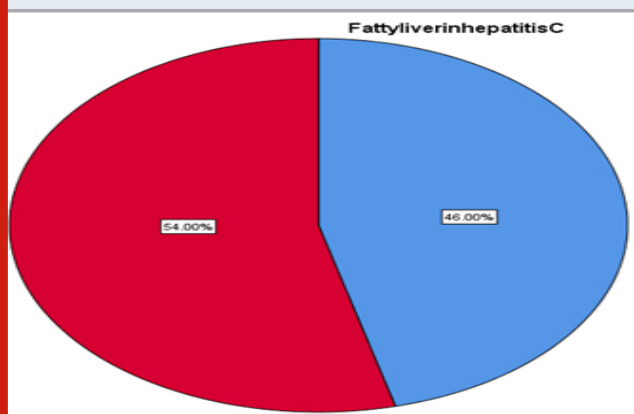

Figure 13: Pie chart represents the percentage of people aware about lifestyle changes cause fatty liver.Red colour in the pie chart denotes"Yes"and blue colour denotes "No". Higher percentage of responses were Yes (72\%).

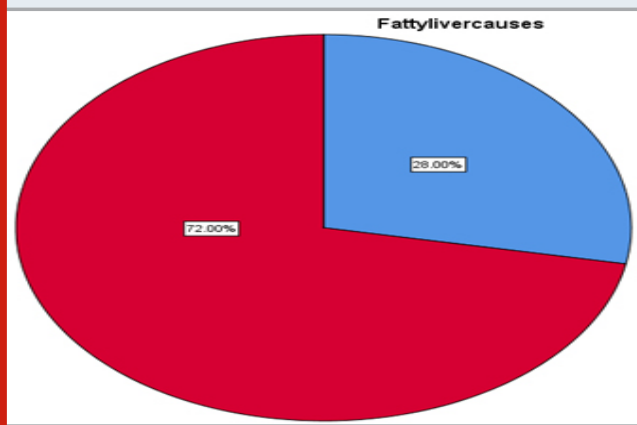

Figure 14: Bar chart showing the comparison of responses based on the question "Do you think fatty liver is caused by lifestyle changes" between male and female. The $\mathrm{X}$ axis represents the sex and Y-axis represents number of participants of which red colour denotes "Yes" and blue colour denotes "No" .Majority of the males thought fatty liver is caused by lifestyle changes.However the difference is not statistically significant (Chi square value - 1.08,p value $=0.298$ ( $>0.5$ - indicating statistically not significant ).

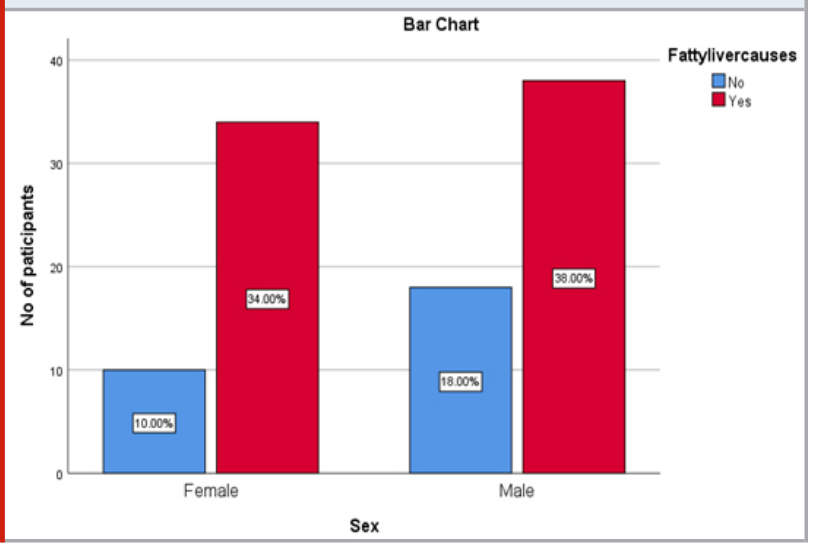

Figure 15: Pie chart represents the percentage of people thought medications cause fatty liver.Red colour in the pie chart denotes"Yes" and blue colour denotes "No". Higher percentage of responses were Yes (60\%).

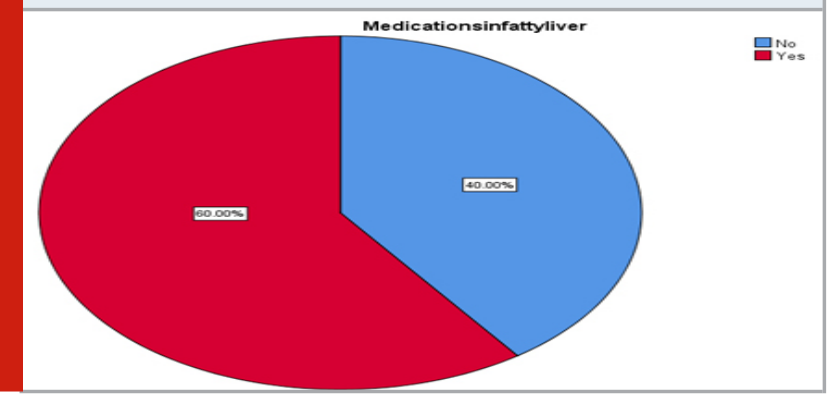

Figure 16: Pie chart represents the percentage of people thought PCOS is linked with fatty liver.Red colour in the pie chart denotes"Yes" and blue colour denotes "No". Higher percentage of responses were Yes (61\%).

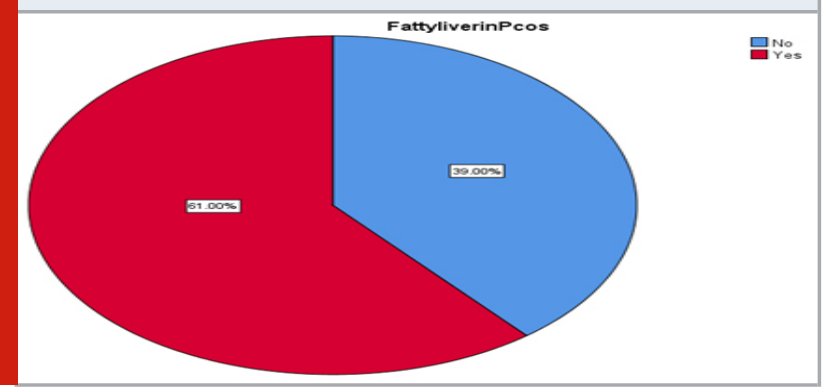

Figure 17: Bar chart showing the comparison of responses based on the question "Do you think fatty liver is linked with PCOS" between male and female. The $\mathrm{X}$ axis represents the sex and Y-axis represents number of participants of which red colour denotes "Yes" and blue colour denotes "No" .Majority of the males thought fatty liver is linked with PCOS.However the difference is not statistically significant (Chi square value $-2.56, \mathrm{p}$ value $=$ 0.113 ( > 0.5 - indicating statistically not significant).

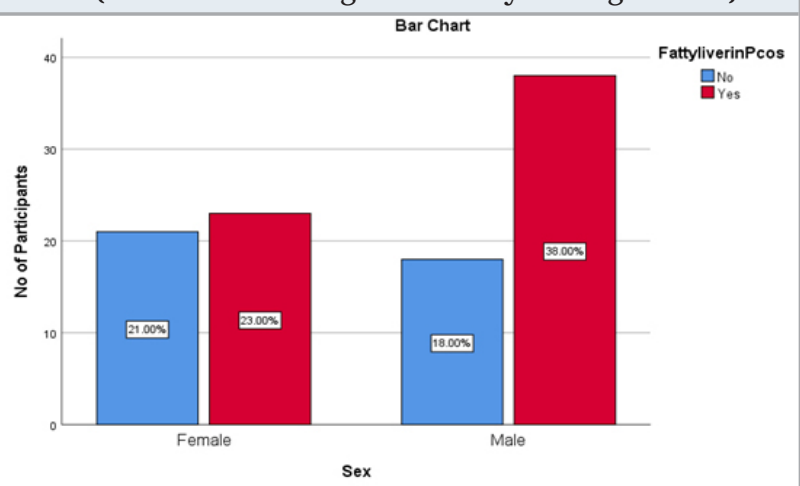


Figure 18: Pie chart represents the percentage of people thought malnutrition causes fatty liver.Red colour in the pie chart denotes"Yes"and blue colour denotes "No". Higher percentage of responses were Yes (60\%).

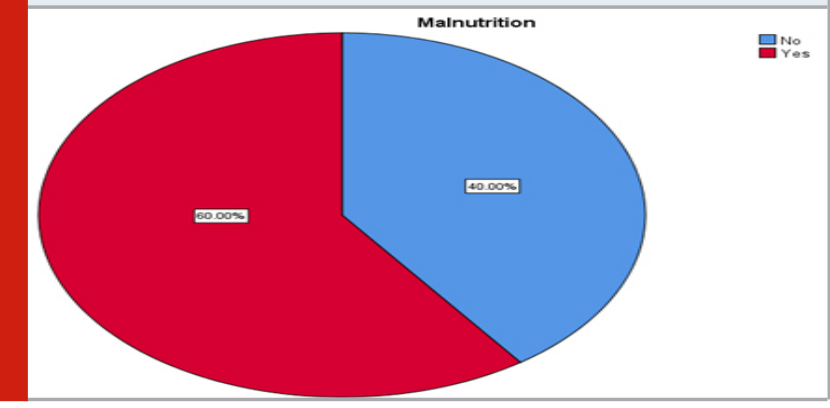

CONCLUSION

Within the limitations of the study we concluded that a majority of the males are more aware about fatty liver than females and also we found that fatty liver diseases are caused by lifestyle changes, malnutrition and it can occur in nonalcoholic people.

\section{ACKNOWLEDGEMENTS}

The authors like to thank the participants of this survey for spending their valuable time.

Conflict of Interest: None declared

\section{REFERENCES}

Angulo, P. (2002) 'Nonalcoholic Fatty Liver Disease', New England Journal of Medicine, pp. 1221-1231. doi: 10.1056/nejmra011775.

Basra, S. and Anand, B. S. (2011) 'Definition, epidemiology, and magnitude of alcoholic hepatitis', World journal of hepatology, 3(5), pp. 108-113.

Bayard, M., Holt, J. and Boroughs, E. (2006) 'Nonalcoholic fatty liver disease', American family physician, 73(11), pp. 1961-1968.

Bedogni, G. et al. (2005) 'Prevalence of and risk factors for nonalcoholic fatty liver disease: The Dionysos nutrition and liver study', Hepatology, pp. 44-52. doi: 10.1002/hep.20734.

Brown, A. J. et al. (2005) 'Polycystic ovary syndrome and severe nonalcoholic steatohepatitis: beneficial effect of modest weight loss and exercise on liver biopsy findings', Endocrine practice: official journal of the American College of Endocrinology and the American Association of Clinical Endocrinologists, 11(5), pp. 319-324.

Caballería, L. et al. (2008) 'Risk factors associated with non-alcoholic fatty liver disease in subjects from primary care units. A case-control study', BMC gastroenterology, 8, p. 44. management of nonalcoholic fatty liver disease: Practice guidance from the American Association for the Study of Liver Diseases', Hepatology, pp. 328-357. doi: 10.1002/hep.29367.

Dyson, J. K., Anstee, Q. M. and McPherson, S. (2015) 'Republished: Non-alcoholic fatty liver disease: a practical approach to treatment', Postgraduate medical journal, 91(1072), pp. 92-101.

Friedman, S. L. et al. (2018) 'Mechanisms of NAFLD development and therapeutic strategies', Nature Medicine, pp. 908-922. doi: 10.1038/s41591-0180104-9.

$\mathrm{Hu}, \mathrm{X} .-$ Y. et al. (2018) 'Risk factors and biomarkers of non-alcoholic fatty liver disease: an observational cross-sectional population survey', BMJ Open, 8(4), p. e019974.

Kawaguchi, T. et al. (2012) 'Genetic polymorphisms of the human PNPLA3 gene are strongly associated with severity of non-alcoholic fatty liver disease in Japanese', PloS one, 7(6), p. e38322.

Kneeman, J. M., Misdraji, J. and Corey, K. E. (2012) 'Secondary causes of nonalcoholic fatty liver disease', Therapeutic advances in gastroenterology, 5(3), pp. 199-207.

Leermakers, E. T. M. et al. (2015) 'Effects of choline on health across the life course: a systematic review', Nutrition Reviews, 73(8), pp. 500-522.

Lieber, C. S. (2004) 'Alcoholic fatty liver: its pathogenesis and mechanism of progression to inflammation and fibrosis', Alcohol, pp. 9-19. doi: 10.1016/j. alcohol.2004.07.008.

Lu, H. et al. (2009) 'High prevalence of coronary heart disease in type 2 diabetic patients with non-alcoholic fatty liver disease', Archives of medical research, 40(7), pp. 571-575.

Marciano, F., Savoia, M. and Vajro, P. (2016) 'Celiac disease-related hepatic injury: Insights into associated conditions and underlying pathomechanisms', Digestive and liver disease: official journal of the Italian Society of Gastroenterology and the Italian Association for the Study of the Liver, 48(2), pp. 112-119.

'National Institute of Diabetes and Digestive and Kidney Diseases (NIDDK)' (2008) Wiley Encyclopedia of Clinical Trials. doi: 10.1002/9780471462422.eoct468.

Osman, K. A., Osman, M. M. and Ahmed, M. H. (2007) 'Tamoxifen-induced non-alcoholic steatohepatitis: where are we now and where are we going?', Expert opinion on drug safety, 6(1), pp. 1-4.

Osna, N. A., Donohue, T. M., Jr and Kharbanda, K. K. (2017) 'Alcoholic Liver Disease: Pathogenesis and Current Management', Alcohol research: current reviews, 38(2), pp. 147-161.

Pagano, G. (2002) 'Nonalcoholic steatohepatitis, insulin 
resistance, and metabolic syndrome: Further evidence for an etiologic association', Hepatology, pp. 367-372. doi: 10.1053/jhep.2002.30690.

Singh, S. et al. (2015) 'Fibrosis progression in nonalcoholic fatty liver vs nonalcoholic steatohepatitis: a systematic review and meta-analysis of paired-biopsy studies', Clinical gastroenterology and hepatology: the official clinical practice journal of the American Gastroenterological Association, 13(4), pp. 643-54. e1-9; quiz e39-40.

Söderberg, C. et al. (2010) 'Decreased survival of subjects with elevated liver function tests during a 28-year follow-up', Hepatology, 51(2), pp. 595-602.

Thursz, M. et al. (2019) 'Alcohol-related liver disease: Areas of consensus, unmet needs, and opportunities for further study', Journal of hepatology, 70(3), pp. 521-530.

Wong, V. W.-S. et al. (2018) 'Noninvasive biomarkers in NAFLD and NASH - current progress and future promise', Nature Reviews Gastroenterology \& Hepatology, pp. 461-478. doi: 10.1038/s41575-018-0014-9.

Younossi, Z. M. (2019) 'Non-alcoholic fatty liver disease - A global public health perspective', Journal of Hepatology, pp. 531-544. doi: 10.1016/j. jhep.2018.10.033. 